\title{
On an augmented Lagrangian-based preconditioning of Oseen type problems
}

\author{
Xin He, Maya Neytcheva* and Stefano Serra Capizzano ${ }^{\dagger}$
}

\begin{abstract}
The paper deals with a general framework for constructing preconditioners for saddle point matrices, in particular as arising in the discrete linearized Navier-Stokes equations (Oseen's problem). We utilize the so-called augmented Lagrangian approach, where the original linear system of equations is first transformed to an equivalent one, which latter is then solved by a preconditioned iterative solution method.

The matrices in the linear systems, arising after the discretization of Oseen's problem, are of two-by-two block form as are the best known preconditioners for these. In the augmented Lagrangian formulation, a scalar regularization parameter is involved, which strongly influences the quality of the block-preconditioners for the system matrix (referred to as outer), as well as the conditioning and the solution of systems with the resulting pivot block (referred to as inner) which, in the case of large scale numerical simulations has also to be solved using an iterative method. We analyse the impact of the value of the regularization parameter on the convergence of both outer and inner solution methods.

The particular preconditioner used in this work exploits the inverse of the pressure mass matrix. We study the effect of various approximations of that inverse on the performance of the preconditioners, in particular that of a sparse approximate inverse, computed in an element-by-element fashion. We analyse and compare the spectra of the preconditioned matrices for the different approximations and show that the resulting preconditioner is independent of problem, discretization and method parameters, namely, viscosity, mesh size, mesh anisotropy.

We also discuss possible approaches to solve the modified pivot matrix block.

Keywords: Navier-Stokes equations, saddle point systems, augmented Lagrangian, finite elements, approximation of mass matrixiterative methods, preconditioning
\end{abstract}

\section{Introduction}

The motivation for this work is to enable fast and reliable numerical simulations of multiphase flow problems, based on a coupled system consisting of the Cahn-Hilliard and Navier-Stokes

\footnotetext{
*Department of Information Technology, Uppsala University, Sweden, he . xin, maya . neyt cheva@it . uu . se

${ }^{\dagger}$ Department of Physics and Mathematics, University of Insubria, Como, Italy, s. serracapizzano@uninsubria. it
} 
(N-S) equations. The development of phase interfaces (concentration profiles), governed by the Cahn-Hilliard equation, is affected by convective processes, the velocities of which are determined by solving the N-S equations. Therefore, efficient solution methods for the discrete $\mathrm{N}-\mathrm{S}$ equations are important.

The complete numerical simulation requires to solve time-dependent N-S. For the purpose of this study, however, we consider only the steady-state form of the N-S problem, which is a linearization of the full problem, based on Picard iterations, comprising a sequence of approximate solutions of the linear Oseen's problem (cf.,for instance [18]). Since the solution of Oseen's problem is a major computational block in the numerical procedure, finding efficient solution methods for Oseen's problem is crucial.

Oseen's problem is formulated as follows.

Let $\Omega \subset \mathbb{R}^{d}(d=2,3)$ be a bounded, connected domain with boundary $\partial \Omega$. Given a force field $\mathbf{f}: \Omega \rightarrow \mathbb{R}^{d}$, at each Picard iteration, find a velocity field $\mathbf{u}: \Omega \rightarrow \mathbb{R}^{d}$ and a pressure field $p: \Omega \rightarrow \mathbb{R}$ such that

$$
\begin{array}{rlrl}
-v \Delta \mathbf{u}+(\mathbf{w} \cdot \nabla) \mathbf{u}+\nabla p & =\mathbf{f} & & \text { in } \Omega, \\
\operatorname{div} \mathbf{u}=0 & & \text { in } \Omega,
\end{array}
$$

subject to suitable boundary conditions on $\partial \Omega$. Here $v>0$ is the kinematic viscosity coefficient, assumed to be constant, (related to the Reynolds number $R_{e}$ as $R_{e}=\frac{\mathrm{UL}}{v}$, where L denotes a characteristic length scale for the domain $\Omega$ and $U$ is some reference value of the velocity). The operator $\Delta$ is the Laplace operator in $\mathbb{R}^{d}, \nabla$ denotes the gradient, and $d i v$ is the divergence operator. The first equation in (1) represents the conservation of momentum, while the second describes the incompressibility condition. The (wind) vector $\mathbf{w}$ is an approximation of the velocity at a previous iteration step, so it is updated at every nonlinear iteration. The vector field $\mathbf{w}$ is considered to be known and divergence free. The linear system arising after discretizing the system (1) is of two-by-two block form

$$
\left[\begin{array}{cc}
A & B^{T} \\
B & O
\end{array}\right]\left[\begin{array}{l}
\mathbf{u}_{h} \\
\mathbf{p}_{h}
\end{array}\right]=\left[\begin{array}{l}
\mathbf{f} \\
\mathbf{g}
\end{array}\right] \quad \text { or } \quad \mathscr{A} \mathbf{x}=\mathbf{b}
$$

where $\mathscr{A}=\left[\begin{array}{cc}A & B^{T} \\ B & O\end{array}\right], \mathbf{u}_{h}$ is the discrete velocities vector, $\mathbf{p}_{h}$ is the discrete pressure vector and $\mathbf{x}^{T}=\left[\begin{array}{ll}\mathbf{u}_{h}^{T} & \mathbf{p}_{h}^{T}\end{array}\right]$. The matrix $A$ is the discretized convection-diffusion operator. The matrix $B$ is the (negative) divergence matrix and $B^{T}$ is the gradient matrix.

Linear systems of the form (2) are referred to as saddle point systems. In recent years developing efficient preconditioning techniques to systems of this form has attracted much attention and the amount of the literature on the topic is vast. We refer to [7], [15], [30], [6] and [18] as more closely related to the present paper.

The matrix $\mathscr{A}$ is of two-by-two block form. The best known preconditioners for such matrices, or more generally $\left[\begin{array}{ll}A_{11} & A_{12} \\ A_{21} & A_{22}\end{array}\right]$, utilize the available block structure and are based on some approximation of the exact block-matrix factorization

$$
\left[\begin{array}{ll}
A_{11} & A_{12} \\
A_{21} & A_{22}
\end{array}\right]=\left[\begin{array}{cc}
I_{1} & 0 \\
A_{21} A_{11}^{-1} & I_{2}
\end{array}\right]\left[\begin{array}{cc}
A_{11} & 0 \\
0 & S_{2}
\end{array}\right]\left[\begin{array}{cc}
I_{1} & A_{11}^{-1} A_{12} \\
0 & I_{2}
\end{array}\right],
$$


where $I_{1}$ and $I_{2}$ are identity matrices of proper dimensions, $A_{11}$ is assumed to be nonsingular and $S_{2}=A_{22}-A_{21} A_{11}^{-1} A_{12}$ is the Schur complement matrix.

The most often used preconditioners for $\mathscr{A}$ are either of full block-factorized form, such as (3) or of block lower- or upper-triangular form, as in (4).

$$
\begin{gathered}
\mathscr{M}_{F}=\left[\begin{array}{cc}
\widetilde{A} & O \\
B & S
\end{array}\right]\left[\begin{array}{cc}
I_{1} & \widetilde{A}^{-1} B^{T} \\
O & I_{2}
\end{array}\right], \\
\mathscr{M}_{L}=\left[\begin{array}{cc}
\widetilde{A} & O \\
B & S
\end{array}\right], \quad \mathscr{M}_{U}=\left[\begin{array}{cc}
\widetilde{A} & B^{T} \\
0 & S
\end{array}\right] .
\end{gathered}
$$

In (3) and (4), $\widetilde{A}$ and $S$ are some approximations of the pivot block $A$ and the Schur complement of $\mathscr{A}, S_{\mathscr{A}}=-B A^{-1} B^{T}$, correspondingly.

The above block preconditioners should obey the well-known requirements, namely, to improve the condition number of the original matrix, to be easy to construct and solve systems with, and also to be well-parallelizable.

From (3) and (4) it is easily seen that the quality of the preconditioner highly depends on how well $A$ and $S_{\mathscr{A}}$ are approximated. In the case of constant viscosity the matrix $A$ is of blockdiagonal form and each block corresponds to a discrete scalar convection-diffusion operator. Experience shows that $A$ can be approximated to an arbitrary accuracy, for example, by using an inner solution method with an optimal order preconditioner, such as a geometric or algebraic Multigrid (MG or AMG) or some other multilevel technique. A much more difficult task, however, is how to construct a numerically and computationally efficient approximation of $S_{\mathscr{A}}$, which is in general dense and it is not practical to form it explicitly. Schur complement approximations for the Stokes and Oseen's problem have been studied very intensively during the past decade. For those problems, high quality approximations of $S_{\mathscr{A}}$ or $S_{\mathscr{A}}^{-1}$ have been constructed, in particular, based on the properties of the underlying differential operators. We refer to [18] and the references therein, and also to [30] for some more theoretical details and numerical studies.

In general, the above mentioned (problem-dependent) preconditioners show convergence behavior, which is mesh-independent. Some of the variants exhibit good convergence properties even for quite small values of the viscosity. At the same time, the suggested approximations of $S_{\mathscr{A}}$ or $S_{\mathscr{A}}^{-1}$ can be costly to apply and construct, or may not be fully robust with respect to mesh size and viscosity. Below we mention a few examples of such preconditioners.

(1) Pressure mass matrix $M_{p}$ A well-established theory and practice suggests to approximate the product matrix $B A^{-1} B^{T}$ by the pressure mass matrix $M_{p}$. Clearly, to compute $M_{p}$ is cheap and the corresponding preconditioner does not depend on the discretization parameter. The matrix $M_{p}$ can be used for Stokes problem and for relatively 'mild' values of $v$ (see, e.g., [28]) but it is not efficient for more general problems. We note also that $M_{p}$ is always symmetric and positive definite while $S_{\mathscr{A}}$ is in general nonsymmetric.

(2) The pressure convection-diffusion (PCD) preconditioner $S_{P C D}$

This preconditioner is first suggested in [23] and is an approximation of $S_{\mathscr{A}}^{-1}$ of the form

$$
S_{P C D}^{-1}=\widetilde{M}_{p}^{-1} A_{p} L_{p}^{-1},
$$


where $A_{p}$ and $L_{p}$ are the pressure convection-diffusion and Laplace matrices correspondingly, and $\widetilde{M}_{p}^{-1}$ denotes some approximate solution with $M_{p} . S_{P C D}$ is a significant improvement over $M_{p}$, however its construction is not straightforward and requires some non-physical boundary conditions for $A_{p}$ and $L_{p}$. Furthermore, the convergence rate degrades as $v \rightarrow 0$. See, for instance, [30] for a broader discussion.

(3) The BFBt preconditioner

This preconditioner is also an approximation of $S_{\mathscr{A}}^{-1}$ and is defined as

$$
S_{B F B t}^{-1}=\left(B \widehat{M}_{u}^{-1} B^{T}\right)^{-1} B \widehat{M}_{u}^{-1} A \widehat{M}_{u}^{-1} B^{T}\left(B \widehat{M}_{u}^{-1} B^{T}\right)^{-1}
$$

where $\widehat{M}_{u}$ is a diagonal approximation of the velocity mass matrix $M_{u}$. This preconditioner is suggested in [17]. As is seen from its definition, no artificial boundary conditions have to be set, and the preconditioner is fairly easy to construct. Still, a dependence on $v$ as well as on $h$ is observed for some flow problems. Further, we note that to apply this preconditioner is more costly than the PCD preconditioner. More details on those Schur approximations and variants of them are provided in, for example, [30].

(4) In [28] a very cheap approximation of the Schur complement has been studied, based on the finite element method (FEM) discretization and an element-by-element (EBE) technique. There, again a mesh-independence convergence has been shown, however the degradation of the convergence rate for $v \rightarrow 0$ is significant.

An established way to circumvent the difficulties to approximate the Schur complement of the block matrix $\mathscr{A}$ is to transform it into a new system with the same solution, which is of the form

$$
\widetilde{\mathscr{A}}=\left[\begin{array}{cc}
A+\gamma B^{T} W^{-1} B & B^{T} \\
B & O
\end{array}\right],
$$

where $\gamma>0$ and $W$ are suitable scalar and matrix (method) parameters, correspondingly.

The technique is well-known and is referred to as the augmented Lagrangian (AL) method. For an early analysis of the AL technique, we refer to [2], see also [7]. The method has been analyzed and tested numerically in various studies. In [2] and [15], $W$ is chosen to be the identity matrix. In [8] a good choice for $W$ is shown to be the pressure mass matrix $M_{p}$.

The major pro and con arguments regarding the transformation (5) are that

(i) for large enough values of $\gamma$, the Schur complement $S_{\widetilde{A}}=-B\left(A+\gamma B^{T} W^{-1} B\right)^{-1} B^{T}$ of $\widetilde{\mathscr{A}}$ can be approximated by a symmetric positive definite (spd) matrix, such as $M_{p}$ in, e.g., [8] or even by a multiple of the identity matrix, as shown in [3].

(ii) the difficult part of the method becomes now the solution of systems with the pivot block of $\widetilde{\mathscr{A}}, \widetilde{A}=A+\gamma B^{T} W^{-1} B$, since it is more dense and becomes increasingly ill-conditioned with $\gamma \rightarrow \infty$. In many studies this block is solved by a direct method, which implies also that the block has to be first explicitly computed in order to be factorized. 
In other studies, specific problem-dependent solution methods have been used, for instance, in [8], a special MG method has been derived for the particular matrix blocks in the considered discrete Oseen's equations. In [3] a domain decomposition technique is used.

The organization of the present paper is as follows. In Section 2 we briefly state the problem formulation, recall the augmented Lagrangian method and analyse the impact of the choice of $\gamma$ on both the solution of systems with $\widetilde{\mathscr{A}}$ and that of systems with $\widetilde{A}$. In Section 3 we consider various approximations of the inverse of the mass matrix for problems discretized using FEM. We analyse and compare the corresponding spectrum of the preconditioned matrices, also related to the method parameter $\gamma$. In Section 4 we discuss the solution of the modified pivot block $\widetilde{A}$ and propose to compute its inverse using the Inverse Sherman-Morrison algorithm from [12, 13]. Section 5 contains numerical illustrations. Some conclusions are given in Section 6.

\section{General setting and preliminaries}

\subsection{Discrete Oseen's problem}

For completeness, we state the FE formulation of the system (1). The standard weak formulation of (1) reads as following (c.f., e.g., [18]):

Let $\mathbf{H}_{E}^{1}=\left\{\mathbf{u} \in \mathscr{H}^{1}(\Omega)^{d} \mid \mathbf{u}=\mathbf{w}\right.$ on $\left.\partial \Omega_{D}\right\}$ and $H_{E_{0}}^{1}=\left\{\mathbf{v} \in \mathscr{H}^{1}(\Omega)^{d} \mid \mathbf{v}=\mathbf{0}\right.$ on $\left.\partial \Omega_{D}\right\}$.

Find $\mathbf{u} \in \mathbf{H}_{E}^{1}$ and $p \in L_{2}(\Omega)$ such that

$$
\begin{array}{r}
v \int_{\Omega} \nabla \mathbf{u}: \nabla \mathbf{v}+\int_{\Omega}(\mathbf{w} \cdot \nabla \mathbf{u}) \cdot \mathbf{v}-\int_{\Omega} p(\nabla \cdot \mathbf{v})=\int_{\Omega} \mathbf{f} \cdot \mathbf{v} \text { for all } \mathbf{v} \in \mathbf{H}_{E_{0}}^{1}, \\
\int_{\Omega} q(\nabla \cdot \mathbf{u})=0 \text { for all } q \in L_{2}(\Omega) .
\end{array}
$$

The convection term can be described by a trilinear form $c: \mathbf{V}_{E_{0}} \times \mathbf{H}_{E}^{1} \times \mathbf{H}_{E_{0}}^{1} \rightarrow \mathbb{R}, c(\mathbf{z} ; \mathbf{u}, \mathbf{v}):=$ $\int_{\Omega}(\mathbf{z} \cdot \nabla \mathbf{u}) \cdot \mathbf{v}$, where the subspace of divergence-free velocities is given by $\mathbf{V}_{E_{0}}:=\left\{\mathbf{z} \in \mathbf{H}_{E_{0}}^{1} ; \nabla\right.$. $\mathbf{z}=0$ in $\Omega\}$. We also introduce the continuous bilinear forms $a: \mathbf{H}^{1} \times \mathbf{H}^{1} \rightarrow \mathbb{R}$ and $b: \mathbf{H}^{1} \times$ $L_{2}(\Omega) \rightarrow \mathbb{R}$ as

$$
a(\mathbf{u}, \mathbf{v}):=v \int_{\Omega} \nabla \mathbf{u}: \nabla \mathbf{v}, \quad \text { and } \quad \mathrm{b}(\mathbf{v}, \mathrm{q}):=-\int_{\Omega} \mathrm{q}(\nabla \cdot \mathbf{v})
$$

Then, the system (6) can be rewritten in terms of bilinear forms, namely, find $\mathbf{u} \in \mathbf{H}_{E}^{1}$ and $p \in L_{2}(\Omega)$ such that

$$
\begin{array}{r}
a(\mathbf{u}, \mathbf{v})+c(\mathbf{w} ; \mathbf{u}, \mathbf{v})+b(\mathbf{v}, p)=\ell(\mathbf{v}) \quad \text { for all } \mathbf{v} \in \mathbf{H}_{E_{0}}^{1} \\
b(\mathbf{u}, q)=0 \quad \text { for all } q \in L_{2}(\Omega)
\end{array}
$$

where $\mathbf{w} \in \mathbf{V}_{E_{0}}$ and $\ell(\mathbf{v}):=\int_{\Omega} \mathbf{f} \cdot \mathbf{v}$ is a continuous function $\ell: \mathbf{H}^{1} \rightarrow \mathbb{R}$. 
The discrete version of (7) reads:

Find $\mathbf{u}_{h} \in \mathbf{X}_{E}^{h}$ and $\mathbf{p}_{h} \in P^{h} \subset L_{2}(\Omega)$ such that

$$
\begin{aligned}
a\left(\mathbf{u}_{h}, \mathbf{v}_{h}\right)+c\left(\mathbf{w}_{h} ; \mathbf{u}_{h}, \mathbf{v}_{h}\right)+b\left(\mathbf{v}_{h}, \mathbf{p}_{h}\right) & =\ell\left(\mathbf{v}_{h}\right) \quad \text { for all } \mathbf{v}_{h} \in \mathbf{V}_{0}^{h} \subset \mathbf{H}_{E_{0}}^{1}, \\
b\left(\mathbf{u}_{h}, \mathbf{q}_{h}\right) & =0 \text { for all } \mathbf{q}_{h} \in P^{h} \subset L_{2}(\Omega) .
\end{aligned}
$$

Here we assume that the FEM discretization satisfies the discrete LBB condition (cf., e.g., [18]).

\subsection{The augmented Lagrangian method}

As already stated above, the augmented Lagrangian formulation of (2) is of the form (5), where $\gamma$ and $W$ are method parameters to be chosen.

One can view the modified system (5), obtained in two ways.

(I) The first (classical) approach is, instead of the original problem (2), to consider a regularized one,

$$
\left[\begin{array}{cc}
A & B^{T} \\
B & -\frac{1}{\gamma} W
\end{array}\right]\left[\begin{array}{l}
\mathbf{u}_{h} \\
\mathbf{p}_{h}
\end{array}\right]=\left[\begin{array}{l}
\mathbf{f} \\
\mathbf{g}
\end{array}\right] \quad \text { or } \quad \overline{\mathscr{A}} \mathbf{x}=\mathbf{b}
$$

for some large enough scalar parameter $\gamma$ and some spd matrix $W$. Systems of this form are obtained via various stabilization techniques. Depending on the stabilization, the system (9) may or may not be consistent with (2), however, for $\gamma \rightarrow \infty$ the modified system converges to the original one, so, clearly, (i) the technique is expected to work well for large values of $\gamma$ and (ii) we can use the form (9) to construct a preconditioner for the matrix in (2).

Based on (9) and the exact factorization of $\overline{\mathscr{A}}$,

$$
\overline{\mathscr{A}}=\left[\begin{array}{cc}
A & B^{T} \\
B & -\frac{1}{\gamma} W
\end{array}\right]=\left[\begin{array}{cc}
I_{1} & -\gamma B^{T} W^{-1} \\
0 & I_{2}
\end{array}\right]\left[\begin{array}{cc}
A+\gamma B^{T} W^{-1} B & 0 \\
0 & -\frac{1}{\gamma} W
\end{array}\right]\left[\begin{array}{cc}
I_{1} & 0 \\
-\gamma W^{-1} B & I_{2}
\end{array}\right]
$$

we see, for example, that

$$
\mathscr{M}_{L}=\left[\begin{array}{cc}
A+\gamma B^{T} W^{-1} B & 0 \\
B & -\frac{1}{\gamma} W
\end{array}\right] \quad \text { or } \quad \mathscr{M}_{U}=\left[\begin{array}{cc}
A+\gamma B^{T} W^{-1} B & B^{T} \\
0 & -\frac{1}{\gamma} W
\end{array}\right]
$$

are good candidates to precondition the matrix $\overline{\mathscr{A}}$ in (9) and, for large values of $\gamma$, for $\mathscr{A}$ itself.

(II) The second approach is to transform (2) algebraically into an equivalent system

$$
\left[\begin{array}{cc}
A+\gamma B^{T} W^{-1} B & B^{T} \\
B & 0
\end{array}\right]\left[\begin{array}{l}
\mathbf{u}_{h} \\
\mathbf{p}_{h}
\end{array}\right]=\left[\begin{array}{l}
\widehat{\mathbf{f}} \\
\mathbf{g}
\end{array}\right]
$$

where $\widehat{\mathbf{f}}=\mathbf{f}+\gamma B^{T} W^{-1} B \mathbf{g}$ (clearly, when $\mathbf{g}=\mathbf{0}$, then $\widehat{\mathbf{f}}=\mathbf{f}$ ).

We note that while in the first approach it is clear that $\gamma$ is supposed to be large and the matrix $W$ is often related to the stabilization method, the transformation (11) holds for any value of $\gamma$, including $\gamma=1$ or $\gamma \ll 1$, and there is more freedom in choosing the (nonsingular) matrix $W$. 
Consider next the matrix $\widetilde{\mathscr{A}}$ from (5) preconditioned by $\mathscr{M}_{L}$, i.e., consider the generalized eigenvalue problem

$$
\widetilde{\mathscr{A}} \mathbf{v}=\lambda \mathscr{M}_{L} \mathbf{v}
$$

We see that

$$
\mathscr{M}_{L}^{-1} \widetilde{\mathscr{A}}=\left[\begin{array}{cc}
I & \left(A+\gamma B^{T} W^{-1} B\right)^{-1} B^{T} \\
0 & \gamma W^{-1} B\left(A+\gamma B^{T} W^{-1} B\right)^{-1} B^{T}
\end{array}\right] .
$$

A calculation, in which we apply Sherman-Morrison-Woodbury's formula to $\left(A+\gamma B^{T} W^{-1} B\right)^{-1}$, reveals that

$$
\gamma W^{-1} B\left(A+\gamma B^{T} W^{-1} B\right)^{-1} B^{T}=\gamma Q-\gamma Q(I+\gamma Q)^{-1} \gamma Q,
$$

where $Q=W^{-1} B A^{-1} B^{T}$. Thus, we see that the eigenvalues $\lambda$ of (12) are equal to 1 (with multiplicity equal to the dimension of $A$ ) and the rest coincide with those of the matrix $\gamma Q-$ $\gamma Q(I+\gamma Q)^{-1} \gamma Q$, i.e., are tightly related to the eigenvalues of $W^{-1} B A^{-1} B^{T}$, where the product $B A^{-1} B^{T}$ is the negative Schur complement of the original saddle point system matrix $\mathscr{A}$ in (2). The following result holds.

Lemma 2.1 Let the matrices $\widetilde{\mathscr{A}}$ and $\mathscr{M}_{L}$ are defined as in (5) and (10), and let $\mu=a+i b$ be any eigenvalue of $B A^{-1} B^{T} \mathbf{w}=\mu W \mathbf{w}$. Let $\delta$ be an eigenvalue of the matrix $\widetilde{Q} \equiv \gamma Q-\gamma Q(I+$ $\gamma Q)^{-1} \gamma Q$, where $Q=W^{-1} B A^{-1} B^{T}$. Then the following holds:

(1) The matrices $Q$ and $\widetilde{Q}$ have the same eigenvectors and the eigenvalues of $\widetilde{Q}$ are equal to

$$
\delta=\frac{\gamma \mu}{1+\gamma \mu}=\frac{1}{1+\frac{1}{\gamma \mu}} .
$$

When $\gamma \rightarrow \infty$ all nonzero eigenvalues of the eigenproblem (12) converge to 1 .

(2) If $\mu=a+i b$ are bounded, then the same holds for $\lambda$. Furthermore, if $a>0$ then there holds

$$
\mathscr{R}(\lambda) \leq 1 \quad \text { and } \quad|\mathscr{I}(\lambda)| \leq \frac{\gamma|b|}{(1+\gamma a)^{2}+(\gamma b)^{2}}
$$

where $\mathscr{R}(\lambda)$ and $\mathscr{I}(\lambda)$ are the real and imaginary part of the eigenvalues $\lambda$ of the generalized eigenvalue (12).

Proof. Part (1) and (2) follow from the expression for $\widetilde{Q}$ and straightforward computations. Using (14), we see that the eigenvalues $\lambda$ take the following form

$$
\lambda=\frac{(1+\gamma a) \gamma a+(\gamma b)^{2}}{(1+\gamma a)^{2}+(\gamma b)^{2}}+i \frac{\gamma b}{(1+\gamma a)^{2}+(\gamma b)^{2}}
$$

Further, if $a>0$, and since $\gamma>0$, we have

$$
\lambda=\frac{\gamma \mu}{1+\gamma \mu}=\frac{(1+a \gamma) a \gamma+b^{2}}{(1+a \gamma)^{2}+b^{2}}+i \frac{b \gamma}{(1+a \gamma)^{2}+b^{2}},
$$


thus, the bounds in (15) hold true.

In earlier work, for example, in [16], it is shown that for $W=M_{p}, \mu$ are contained in a rectangle in $\mathscr{R}(z)>0$. In Lemma 2.2 we generalize the latter result for any positive definite matrix $W$.

Lemma 2.2 Consider matrices $A$ of size $n \times n, B$ of size $m \times n$, and $W$ of size $m \times m$, where the symmetric part of $A, \mathscr{S}(A)=\left(A+A^{*}\right) / 2$ and $W$ are positive definite. Let $\mu$ be any eigenvalue of $Q=W^{-1} B A^{-1} B^{T}$ and assume $B$ full rank with $n \geq m$. Then $\mathscr{R}(\mu)>0$ (i.e. the eigenvalues of $Q$ lie in a positive box).

Proof. For any square matrix $X$, when $\mathscr{S}(X)$ is positive definite, it is straightforward to check that its field of values $\mathscr{F}(X)=\left\{\mathbf{f}^{*} X \mathbf{f}, \mathbf{f} \in C^{n}, \mathbf{f}^{*} \mathbf{f}=1\right\}$ is contained in the strictly positive half complex plane and, therefore, the eigenvalues of $X$, being a subset of $\mathscr{F}(X)$, have positive real parts. To prove that $\mathscr{R}(\mu)>0$ it suffices to observe that $Q$ and $\widetilde{Q}$ are spectrally equivalent, $\widetilde{Q}=W^{-1 / 2} B A^{-1} B^{T} W^{-1 / 2}$ and to show that $\widetilde{Q}$ has also a positive definite symmetric part. . To prove that $\mathscr{S}(\widetilde{Q})$ is positive definite, we first observe that $\mathbf{f}^{*} \widetilde{Q} \mathbf{f}=\mathbf{g}^{*} A^{-1} \mathbf{g}$, where $\mathbf{g}=B^{T} W^{-1 / 2} \mathbf{f}$ with $\mathbf{f} \neq \mathbf{0}$ implies that $\mathbf{g}$ is nonzero, because $B$ is full rank and $n \geq m$. Hence, to show the positive definiteness of $\mathscr{S}(\widetilde{Q})$ it suffices to demonstrate the positive definiteness of $\mathscr{S}\left(A^{-1}\right)$. The latter is trivial, since

$$
\mathscr{S}(A)=\left(A+A^{*}\right) / 2
$$

being positive definite, is equivalent to have that the matrix

$$
\left(U^{*}\left(A+A^{*}\right) U\right) / 2
$$

is positive definite for some invertible matrix $U$ (congruence transformation). By choosing $U=$ $A^{-1}$ we obtain $\left(U^{*}\left(A+A^{*}\right) U\right) / 2=\left(A^{-1}+A^{-*}\right) / 2=S\left(A^{-1}\right)$ and the proof is completed.

Next we discuss the solution of systems wit the transformed pivot block $\widetilde{A}=A+\gamma B^{T} W^{-1} B$. In earlier publications, i.e., in [8] and [29], preconditioners for $\widetilde{A}$ are proposed and shown to work efficiently for small values of $\gamma$. We first point out the obvious relation

$$
\widetilde{A}=\left(I+\gamma B^{T} W^{-1} B A^{-1}\right) A
$$

and note that for small $\gamma(\gamma \ll 1)$ the influence of the term $B^{T} W^{-1} B$ is suppressed and $\widetilde{A}$ begins to resemble $A$. Next we show that even though by using the augmented Lagrangian formulation we seem to have avoided the difficulty to approximate the negative Schur complement $B A^{-1} B$, that task still (implicitly) remains. To better clarify the latter claim, we state the next lemma.

Lemma 2.3 Let $X$ and $Y$ are two general matrices, $X \in \mathbb{C}^{n \times m}$ and $Y \in \mathbb{C}^{m \times n}$. Then the spectrum of the products $X Y$ and $Y X$ are identical up to some additional zero eigenvalues of multiplicity $(\max (n, m)-\min (n, m))$.

Proof.

(a) Let $X$ and $Y$ be square, i.e., $n=m$. 
(a1) Let $X$ be nonsingular. Then $X Y$ is spectrally equivalent to $Y X$ because of the trivial equality

$$
X Y=X(Y X) X^{-1} .
$$

Clearly, if $Y$ is nonsingular, then $Y X=Y(X Y) Y^{-1}$ and the result holds true again.

(a2) Let both $X$ and $Y$ be singular. We consider the Schur decomposition of $X, X=U T U^{*}$, where $U$ is unitary and $T$ is upper triangular with diagonal elements equal to the eigenvalues of $X$. Clearly, at least one diagonal entry in $T$ is zero. Let $\varepsilon>0$ and construct $T_{\varepsilon}$ as following

$$
\left(T_{\varepsilon}\right)_{i j}=\left\{\begin{array}{cc}
T_{i j} & \text { for } i \neq j \\
T_{i i} & \text { if } T_{i i} \neq 0 \\
\varepsilon & \text { if } T_{i i}=0
\end{array}\right.
$$

Let $X_{\varepsilon}=U T_{\varepsilon} U^{*}$. Clearly $X_{\varepsilon}$ is nonsingular and $\left\|X-X_{\mathcal{\varepsilon}}\right\|<\varepsilon$.

Due to (a1), there holds that the spectrum of $X_{\varepsilon} Y$ coincides with that of $Y X_{\varepsilon}$ as well as their characteristic polynomials, i.e., $P\left(X_{\varepsilon} Y\right)=P\left(Y X_{\varepsilon}\right)$. Using the fact that the matrices are continuous functions of their entries and so are the coefficients of their characteristic polynomials, letting $\varepsilon \rightarrow 0$ we obtain that

$$
P(X Y)=P(Y X) .
$$

(b) Let now $n \neq m$, and for convenience assume that $n>m$. Augmenting $X$ and $Y$ to square matrices by adding $(n-m)$ columns, respectively rows,

$$
\begin{gathered}
\widetilde{X}=\left[\begin{array}{cc}
X & 0
\end{array}\right], \widetilde{Y}=\left[\begin{array}{l}
Y \\
0
\end{array}\right], \\
\widetilde{X} \widetilde{Y}=X Y, \\
\widetilde{Y} \widetilde{X}=\left[\begin{array}{l}
Y \\
0
\end{array}\right]\left[\begin{array}{ll}
X & 0
\end{array}\right]=\left[\begin{array}{cc}
Y X & 0 \\
0 & 0
\end{array}\right] .
\end{gathered}
$$

By (i2) we know that the spectrum of $\widetilde{X} \widetilde{Y}$ is equal to $\widetilde{Y} \widetilde{X}$, which in its turn coincides with the spectrum of $Y X$ augmented with $(n-m)$ zero eigenvalues.

In [8] and [29], it is observed numerically and noted that the choice $W=M_{p}$ and $\gamma=1$ suffices to obtain a high quality AL-type preconditioner for Oseen's problem. Further, in [11, 9], preconditioners for $A+\gamma B^{T} W^{-1} B$ are derived, which work well for relatively small values of $\gamma$. Here we explain the above behaviour via a more general framework, based on the results in Lemma 2.1, 2.2, 2.3 and (14).

Lemma 2.2 states that if the eigenvalues of $A^{-1}$ (i.e., of $A$ ) are bounded, then those of $Q=$ $W^{-1} B A^{-1} B^{T}$ are also bounded. Denote the eigenvalues of $Q$ by $\mu$, i.e.,

$$
B A^{-1} B^{T} \mathbf{v}=\mu W \mathbf{v}
$$




\begin{tabular}{ccccc}
\hline Size & $\gamma=v$ & $\gamma=1$ & $\gamma=1 / v$ & $\gamma=1000$ \\
\hline \multicolumn{5}{c}{$v=1$} \\
\hline 578 & $15(10)$ & $15(10)$ & $15(10)$ & $2(42)$ \\
2178 & $15(10)$ & $15(10)$ & $15(10)$ & $2(51)$ \\
8450 & $15(11)$ & $15(11)$ & $15(11)$ & $2(53)$ \\
\hline \multicolumn{5}{c}{$v=0.1$} \\
\hline 578 & $28(3)$ & $16(11)$ & $4(38)$ & $2(54)$ \\
2178 & $29(3)$ & $15(13)$ & $4(44)$ & $2(66)$ \\
8450 & $26(3)$ & $14(14)$ & $4(45)$ & $2(71)$ \\
\hline \multicolumn{5}{c}{$v=0.01$} \\
\hline 578 & $58(3)$ & $49(11)$ & $5(65)$ & $3(76)$ \\
2178 & $83(3)$ & $47(14)$ & $4(115)$ & $2(135)$ \\
8450 & $106(3)$ & $45(18)$ & $4(154)$ & $2(179)$ \\
\hline
\end{tabular}

Table 1: Outer(inner) iterations as functions of $v$ and $\gamma$

Lemma 2.3 suggests that we could use $A$ to precondition $A+\gamma B^{T} W^{-1} B$. Indeed, the spectrum of $B^{T} W^{-1} B A^{-1}$ coincides with that of $W^{-1} B A^{-1} B^{T}$ (up to some additional zeros), due to Lemma 2.3 with $X=B^{T}$ and $Y=W^{-1} B A^{-1}$. Thus, the eigenvalues of $\left(A+\gamma B^{T} W^{-1} B\right) A^{-1}$ and those of $A^{-1}\left(A+\gamma B^{T} W^{-1} B\right)$ are equal to $1+\gamma \mu$ or to 1 , so that since $\gamma>0$ and $\mu$ has positive real part, we deduce that the spectral conditioning of $A^{-1}\left(A+\gamma B^{T} W^{-1} B\right)$ is of form

$$
\kappa\left(A^{-1}\left(A+\gamma B^{T} W^{-1} B\right)\right)=|1+\gamma \mu| .
$$

Therefore, given $\mu$, the parameter $\gamma$ controls the inner iterations when solving the transformed pivot block, preconditioned by $A$ itself. Clearly, for a fast convergence, we need $\gamma$ to be as small as possible.

On the other hand, we recall that the number of outer iterations is governed by the spectrum of the preconditioned matrix $\mathscr{M}_{L}^{-1} \widetilde{\mathscr{A}}$, the eigenvalues of which are equal to $1 /(1+1 /(\gamma \mu))$ or to 1 . Again, the positivity of $\gamma$ and of the real part of $\mu$ (the same $\mu$ as in (16)) imply that the spectral conditioning of $\widehat{\mathscr{M}}_{L}^{-1} \widetilde{\mathscr{A}}$ is of the form

$$
\kappa\left(\widehat{\mathscr{A}}^{-1} \widetilde{\mathscr{A}}\right)=|1+1 /(\gamma \mu)|
$$

In other words, due to Lemma 2.3 and (17), we see that in order to guarantee fast convergence of the outer solver (independently of $\mu$ ), $\gamma$ has to be chosen large. Table 1 illustrates the above effects. For various values of $\gamma$ and $v$ we present outer iterations, where the block $A+\gamma B^{T} W^{-1} B$ has been solved with a direct method. As predicted, the outer iteration counts decrease with increasing $\gamma$, independently of $v$, respectively $\mu$. In brackets, we show the number of iterations, needed to solve $\widetilde{A}$, preconditioned by $A$, using full gmres with a relative stopping criterion $10^{-6}$. As expected, small values of $\gamma$ entail fast convergence of the inner method, again independently of $\mu$. 
A natural attempt would be to try to balance the two constraints in (16) and (17), for instance, by minimizing the quantity

$$
\left(|1+\gamma \mu|^{p}+|1+1 / \gamma \mu|^{p}\right)^{1 / p}
$$

which is the $l^{p}$-norm, $p \geq 1$, of the vector with components - the expressions of the spectral conditioning which govern the inner and outer convergence, respectively.

A direct computation shows that, independently of $p, \gamma \mu$ should be $O(1)$ and since we want $\gamma$ to be also $O(1)$, we conclude that $\mu=O(1)$, i.e., $W$ should approximate well the negative Schur complement of the original system, $B A^{-1} B^{T}$. More specifically, the minimum of (18) is attained for real positive $\mu$ and for $p=1$ the global minimum over the positive complex half plane is attained when $\gamma \mu=1$.

The above reasonings, although appearing to be trivial, show that the attempts to approximate the modified pivot block $A+\gamma B^{T} W^{-1} B$ with matrices or computational procedures, which perform reasonably well for small values of $\gamma$, ultimately destroy the outer convergence rate. In other words, the weight of the product $B^{T} W^{-1} B$ is significant and cannot be neglected.

Remark 2.1 Consider the minimization of the expression in (18) and the following more general functional

$$
\phi_{p, \alpha}(z)=\left(|1+z|^{p}+|\alpha+1 / z|^{p}\right)^{1 / p}, \quad z \in \mathbb{C}^{+}, p \geq 1, \alpha>0,
$$

and let $z=\gamma \mu$. Clearly, for $\alpha=1, \phi_{p, \alpha}(z)$ coincides with the functional in (18). The first observation is that for any $y \neq 0$ it holds

$$
\phi_{p, \alpha}(x+i y)=\phi_{p, \alpha}(x-i y)>\phi_{p, \alpha}(x) .
$$

As a consequence, the minimization of $\phi_{p, \alpha}(z)$ for $z \in \mathbb{C}^{+}$reduces to the minimization of $\phi_{p, \alpha}(x)$ for $x \in \mathbb{R}$ which, in its turn, is equivalent to the minimization of $\phi_{p, \alpha}^{p}(x)$ for $x \in \mathbb{R}^{+}$. Since

$$
\frac{d}{d x} \phi_{p, \alpha}^{p}(x)=p\left[(1+x)^{p-1}-(\alpha+1 / x)^{p-1} / x^{2}\right]
$$

it follows that for $p=1$ the minimization point is at $x=1$ independently of $\alpha>0$. Moreover, for $\alpha=1$ the minimization point is again attained at $x=1$ independently of $p \geq 1$. Thus, the parameter $\alpha>0$ does not influence the behaviour of $\phi_{p, \alpha}(z)$. The latter means that preconditioners, which would lead to a coefficient $\alpha \neq 1$ in $\phi_{p, \alpha}(z)$, would still encounter the same problem as discussed above.

\section{On the choice $W=M_{p}$}

While the AL technique is applicable to a general matrix of saddle point form and $W$ can be any spd matrix, for matrices arising from discrete Oseen's (and Stokes) problems, the choice $W=M_{p}$ seems quite natural, as the pressure mass matrix $M_{p}$ is an easily available approximation of the Schur complement and approximates $S_{\mathscr{A}}$ reasonably well, at least for moderately small values of $v$. In what follows we fix $W=M_{p}$. 
Observe, that when solving systems with a preconditioners of the form (10), we need the action of $M_{p}^{-1}$ twice. For certain FEM discretizations, such as when piecewise-constant basis functions are used for the pressure variable, $M_{p}$ is diagonal and then it is trivial to invert it. For other discretizations, a broadly used practice is to replace $M_{p}$ by its diagonal, $D_{M_{p}}$, which turns out to be a very good approximation of $M_{p}$. Spectral bounds for $D_{M_{p}}^{-1} M_{p}$ are derived in [31] for mass matrices arising from various FEM discretizations. For instance, there it is shown that in 2D, for linear FEM on triangles

$$
\frac{1}{2} \leq \lambda\left(D_{M_{p}}^{-1} M_{p}\right) \leq 2, \quad \varkappa\left(D_{M_{p}}^{-1} M_{p}\right) \leq 4
$$

and for bilinear FEM on rectangles

$$
\frac{1}{4} \leq \lambda\left(D_{M_{p}}^{-1} M_{p}\right) \leq \frac{9}{4} \quad \varkappa\left(D_{M_{p}}^{-1} M_{p}\right) \leq 9 .
$$

Another option is to replace $M_{p}$ by the so-called lumped mass matrix

$$
L_{M_{p}}=\operatorname{diag}\left(\ell_{k}\right), \ell_{k}=\sum_{j=1}^{n_{p}} m_{k, j}, k=1, \cdots, n_{p},
$$

where $m_{k, j}$ are the elements of $M_{p}$ and $n_{p}$ is its dimension. Here and in the sequel, $\lambda(A)$ denotes any eigenvalue of a (spd) matrix $A, \lambda_{\min }(A)$ and $\lambda_{\max }(A)$ are the extremal ones and $\varkappa(A)=$ $\lambda_{\text {max }}(A) / \lambda_{\min }(A)$ denotes the spectral condition number.

In the next section we derive another high quality, relatively cheap to construct approximate inverse of $M_{p}$, based on the finite element discretization of the original partial differential equations (1).

\section{The FEM element-by-element sparse approximate inverse of a mass matrix}

Let $A$ be a finite element matrix, obtained via a standard FEM assembly of element matrices $A_{k}$ over a number of finite elements $\left(k=1,2, \cdots, n_{E}\right)$,

$$
A=\sum_{k=1}^{n_{E}} R_{k}^{T} A_{k} R_{k}
$$

Here $R_{k}$ are the standard Boolean matrices which prescribe the local-to-global correspondence of the degrees of freedom. We consider the case when $A$ is spd.

Denote by $n_{e}$ the number of local degrees of freedom per finite element and let $n$ be the number of global degrees of freedom, i.e. $A$ has $\operatorname{order}(n, n)$. Then $R_{k}$ is of size $\left(n_{e}, n\right)$. We recall a technique from [31] to represent $A$ as follows.

Let $R=\left[R_{1} ; R_{2} ; \cdots R_{n_{E}}\right]$ of size $\left(n \cdot n_{e}, n\right)$ and $D_{A}=\operatorname{diag}\left(A_{k}, k=1, \cdots, n_{E}\right)$ of size $\left(n \cdot n_{e}, n \cdot n_{e}\right)$. Then,

$$
A=R^{T} D_{A} R
$$


Based on the representation (20), bounds for the spectrum and the condition number of finite element stiffness, mass and flexibility (the inverse of the stiffness) matrices have been derived in earlier works. We refer, for instance to $[19,20]$ and the references therein, and also to [31].

We now consider constructing an approximate inverse of $A$ of the form

$$
\widehat{A}^{-1}=\sum_{k=1}^{m} R_{k}^{T} A_{k}^{-1} R_{k} .
$$

Below, we refer to this technique as 'EBE-SPAI' to abbreviate 'element-by-element sparse approximate inverse'. To avoid confusion, we point out that EBE-SPAI is not related to other techniques to construct sparse approximate inverses of matrices, also referred to as 'SPAI' such as, for instance, in the works by $[24,22,14,10]$ and others. The major difference is that the classical SPAI techniques construct an approximate inverse relying on a priori determined sparsity structure such as tridiagonal, banded, etc, while EBE-SPAI is only applicable for FEM matrices and uses the sparsity structure prescribed by the underlying discretization mesh.

The EBE-SPAI idea is not new. The technique has been used early in [25] to approximate a Schur complement and to construct an approximate LU factorization of a FEM matrix. In various forms, it has been used, for example, in [27, 4] to approximate in an EBE fashion the inverses of matrix blocks and Schur complements. Since the form of $\widehat{A}^{-1}$ preserves the standard FEM assembly procedure and the element matrices $A_{k}$ are of small dimension and can be analysed locally, the techniques from $[19,31]$ are applicable.

Below we apply the EBE technique to a mass matrix named here with the generic notation $M$. The analysis is additionally simplified by the fact that the mass matrices are symmetric and positive definite (spd).

Consider $M=\sum_{k=1}^{n_{E}} R_{k}^{T} M_{k} R_{k}$ and its EBE-SPAI approximation $\widehat{M}^{-1}=\sum_{k=1}^{n_{E}} R_{k}^{T} M_{k}^{-1} R_{k}$.

Spectral bounds for $M \widehat{M}^{-1}$ can be determined rather straightforwardly. Using the representation (20), and Gershgorin type of arguments as those from [19], we have

$$
\min _{k}\left(\lambda_{\min }\left(M_{k}\right)\right) \leq \lambda(\widehat{M}) \leq p \max _{k}\left(\lambda_{\max }\left(M_{k}\right)\right)
$$

where $p$ the maximum number of finite elements meeting at a nodepoint in the mesh (or the maximum graph degree of the mesh). The spectral bounds in (22) hold true for any matrix obtained via FEM assembly of element matrices (cf. [4]). Further, using the result

$$
\lambda_{\text {min }}(A) \lambda_{\text {min }}(B) \leq \lambda(A B) \leq \lambda_{\max }(A) \lambda_{\max }(B)
$$

which holds for spd matrices (see, e.g., [1], Chapter 3 ) we are in a position to estimate $\lambda\left(\widehat{M}^{-1}\right)$, $\lambda\left(\widehat{M}^{-1} M\right), \lambda\left(D_{\widehat{M}^{-1}} M\right)$ and $\lambda\left(\widehat{M}^{-1} D_{\widehat{M}^{-1}}^{-1}\right)$, where $D_{\widehat{M}^{-1}}$ is the diagonal of $\widehat{M}^{-1}$.

The eigenvalue bounds in (22) are computable and can be quantified for any particular finite element basis functions and for any given discretization mesh. Analogous reasoning can be used to estimate the eigenvalues of $\widehat{M}^{-1}$ via those of the element matrices $M_{k}^{-1}$.

Similarly to the analysis in [31], we show that the factor $p$ in (22) can be omitted, which ensures a convergence independent on mesh size and anisotropy. We quantify the bounds in (23) by two examples. 
Example 3.1 Consider 2D, arbitrary triangular mesh and linear basis functions. In that case, if $\alpha_{k}$ is the area of the $k$ th triangle, then

$$
\begin{aligned}
M_{k} & =\frac{\alpha_{k}}{12}\left[\begin{array}{ccc}
2 & 1 & 1 \\
1 & 2 & 1 \\
1 & 1 & 2
\end{array}\right], & & \lambda\left(M_{k}\right)=\frac{\alpha_{k}}{12}[1,1,4] \\
M_{k}^{-1} & =\frac{3}{\alpha_{k}}\left[\begin{array}{ccc}
3 & -1 & -1 \\
-1 & 3 & -1 \\
-1 & -1 & 3
\end{array}\right], & & \lambda\left(M_{k}^{-1}\right)=\frac{3}{\alpha_{k}}[1,4,4] \\
D_{M_{k}^{-1} M_{k}} & =\frac{3}{4}\left[\begin{array}{ccc}
2 & 1 & 1 \\
1 & 2 & 1 \\
1 & 1 & 2
\end{array}\right], & & \lambda\left(D_{M_{k}^{-1}} M_{k}\right)=\frac{3}{4}[1,1,4] \\
M_{k}^{-1} D_{M_{k}^{-1}}^{-1} & =\frac{1}{3}\left[\begin{array}{ccc}
3 & -1 & -1 \\
-1 & 3 & -1 \\
-1 & -1 & 3
\end{array}\right], & & \lambda\left(M_{k}^{-1} D_{M_{k}^{-1}}^{-1}\right)=\frac{1}{3}[1,4,4] .
\end{aligned}
$$

Then, the following spectral eigenvalue bounds hold:

$$
\frac{1}{3} \leq \lambda\left(\widehat{M}^{-1} D_{\widehat{M}^{-1}}^{-1}\right) \leq \frac{4}{3}, \quad \frac{3}{4} \leq \lambda\left(D_{\widehat{M}^{-1}} M\right) \leq 3
$$

and, using the fact that $\widehat{M}^{-1} M=\widehat{M}^{-1} D_{\widehat{M}^{-1}}^{-1} D_{\widehat{M}^{-1}} M$, we obtain

$$
\frac{1}{4} \leq \lambda\left(\widehat{M}^{-1} M\right) \leq 4 .
$$

Thus, $\varkappa\left(D_{\widehat{M}^{-1}} M\right) \leq 4, \varkappa\left(\widehat{M}^{-1} D_{\widehat{M}^{-1}}^{-1}\right) \leq 4$ and $\varkappa\left(\widehat{M}^{-1} M\right) \leq 16$. Here we have used that all matrices are spd.

Example 3.2 Consider again 2D, a rectangular mesh and bilinear basis functions. In this case, for a rectangular element with sides $h_{2}$ and $h_{y}$ the element mass matrix is the following:

$$
M_{k}=\frac{\alpha_{k}}{36}\left[\begin{array}{cccc}
4 & 2 & 1 & 2 \\
2 & 4 & 2 & 1 \\
1 & 2 & 4 & 2 \\
2 & 1 & 2 & 4
\end{array}\right], \quad \lambda\left(M_{k}\right)=\frac{\alpha_{k}}{36}[1,3,3,9]
$$

and its exact inverse is

$$
M_{k}^{-1}=\frac{9}{\alpha_{k}}\left[\begin{array}{rrrr}
4 & -2 & 1 & -2 \\
-2 & 4 & -2 & 1 \\
1 & -2 & 4 & -2 \\
-2 & 1 & -2 & 4
\end{array}\right], \quad \lambda\left(M_{k}^{-1}\right)=\frac{4}{\alpha_{k}}[1,3,3,9]
$$

Then

$$
\frac{1}{4} \leq \lambda\left(\widehat{M}^{-1} D_{\widehat{M}^{-1}}^{-1}\right) \leq \frac{9}{4} \quad 1 \leq \lambda\left(D_{\widehat{M}^{-1}} M\right) \leq 9
$$


The spectrum bounds become

$$
\frac{1}{4} \leq \lambda\left(\widehat{M}^{-1} M\right) \leq \frac{81}{4}, \quad \varkappa\left(\widehat{M}^{-1} M\right) \leq 81 .
$$

A comparison between estimates (26) and (27) with those in [31] does not indicate any improvement in the approximation of $M^{-1}$ when using $\widehat{M}^{-1}$ instead of $D_{M}^{-1}$. The question why should one use $\widehat{M}^{-1}$ instead of $D_{M}^{-1}$, which latter is much cheaper to compute, is relevant. To answer the question, we note that the difference in the cost to compute $D_{\widehat{M}^{-1}}$ instead of $D_{M}^{-1}$ is the computation of the inverses of the element stiffness matrices only, an operation which is fully parallelizable. Second, we check numerically what the impact is of using $D_{M}^{-1}, L_{M}^{-1}, D_{\widehat{M}^{-1}}$ and $\widehat{M}^{-1}$ on the shape of the spectrum of the preconditioned matrix $\mathscr{M}_{L}^{-1} \widetilde{\mathscr{A}}$, confining ourselves to the FEM setting of Example 3.2.

In Figures 1 and $2(\gamma=1)$ we observe that for $D_{\widehat{M}^{-1}}$ the clustering of the eigenvalues around 1 in the complex plane is best, which also reflects the corresponding number of iterations in Table 2. From the same figures we also see that the clustering is least favourite for $L_{M}^{-1}$. The results do not depend on the value of the viscosity parameter $v$.

As predicted by (14), the clustering of the spectrum is much better for larger values of $\gamma$, as is seen in Figures 3 and $4(\gamma=1000)$. The approximation $D_{\widehat{M}^{-1}}$ is still better than $D_{M}^{-1}$ in the sense that the imaginary part of the spectrum for the former is a magnitude smaller than that of the latter. However, for large values of $\gamma$ the iterations needed to solve the preconditioned system are so few that these can not be further improved, cf. Table 2.

\section{How to solve systems with matrices of the type $A+B^{T} W^{-1} B$ ?}

In the numerical examples, presented so far in this paper, systems with the pivot block $\widetilde{A}$ are solved using a direct method, which is of course not feasible for large scale systems. On the other hand, accurate solutions with $\widetilde{A}$ are crucial for the overall performance of the AL method. In the next section we propose We discuss now briefly a possible approach to solve systems with the modified pivot block of $\widetilde{\mathscr{A}}, \widetilde{A}=A+B^{T} M^{-1} B$, which can be seen as an alternative to the direct method, but could be implemented in a computationally more efficient way.

As is seen from the results in Section 3 , we can safely replace $M^{-1}$ by $D_{M}^{-1}$ or by $\widehat{M}^{-1}$ and, in its turn, $\widehat{M}^{-1}$ - by its diagonal $D_{\widehat{M}^{-1}}$.

Denote $\widehat{B}=\widehat{D}^{-1} B$, where $\widehat{D}^{-1}$ is one of the diagonal matrices $D_{M}^{-1}$ or $D_{\widehat{M}^{-1}}$. The algorithm is, however applicable in more general settings.

We notice that the matrix product $B^{T} \widehat{B}$ can be written as a sum of rank-one matrices,

$$
B^{T} \widehat{B}=\sum_{k=1}^{m} \mathbf{b}_{k} \widehat{\mathbf{b}}_{k}^{T},
$$

where $\mathbf{b}_{k}$ and $\widehat{\mathbf{b}}_{k}$ are the column vectors of $B^{T}$ and $\widehat{B}^{T}$, correspondingly. Further, we represent $A$ as

$$
A=I_{n}+\sum_{k=1}^{n} \mathbf{e}_{k} \widehat{\mathbf{a}}_{k}^{T}
$$




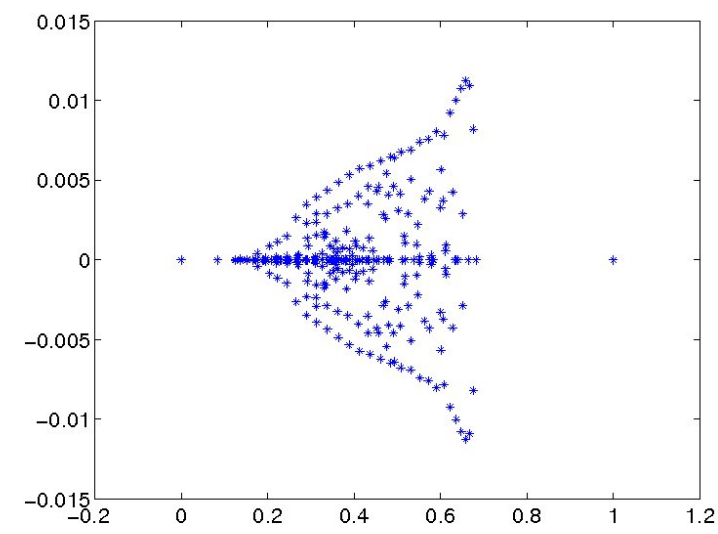

(a) $D_{M}^{-1}$

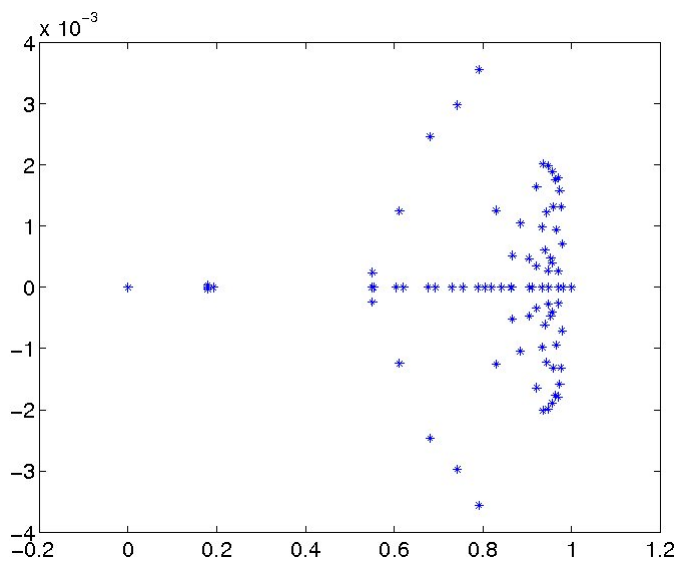

(c) $D_{\widehat{M}^{-1}}$

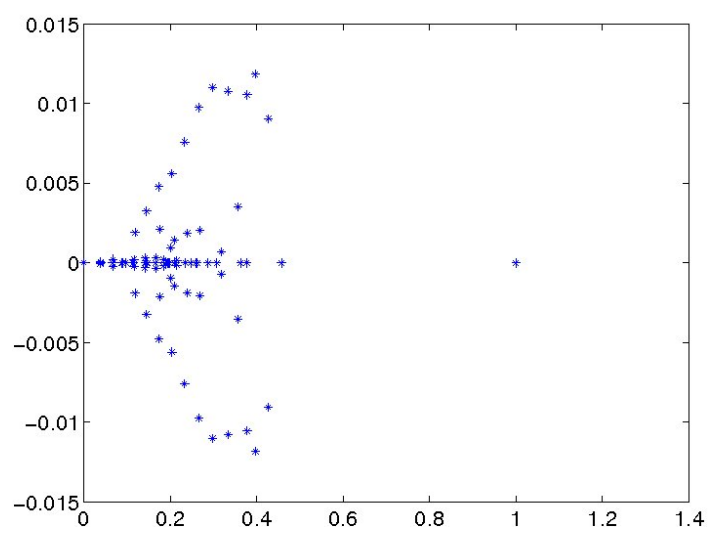

(b) $L_{M}^{-1}$

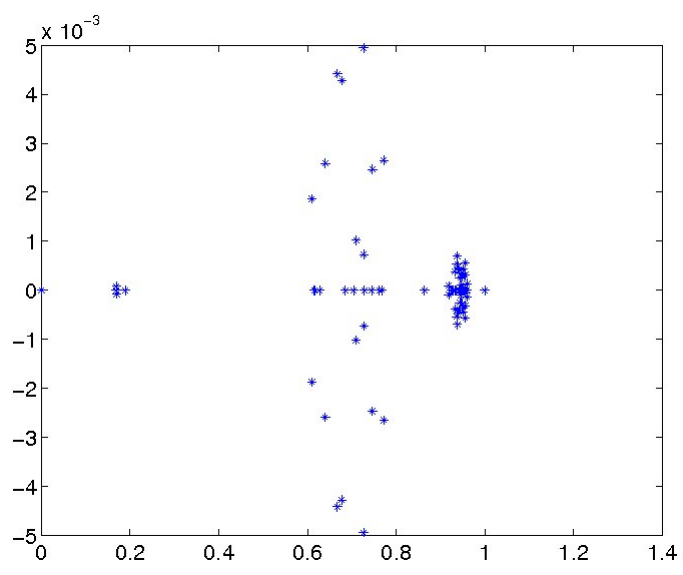

(d) $\widehat{M}^{-1}$

Figure 1: The spectrum of $\mathscr{M}_{L}^{-1} \widetilde{\mathscr{A}}, \gamma=1, v=1$ 


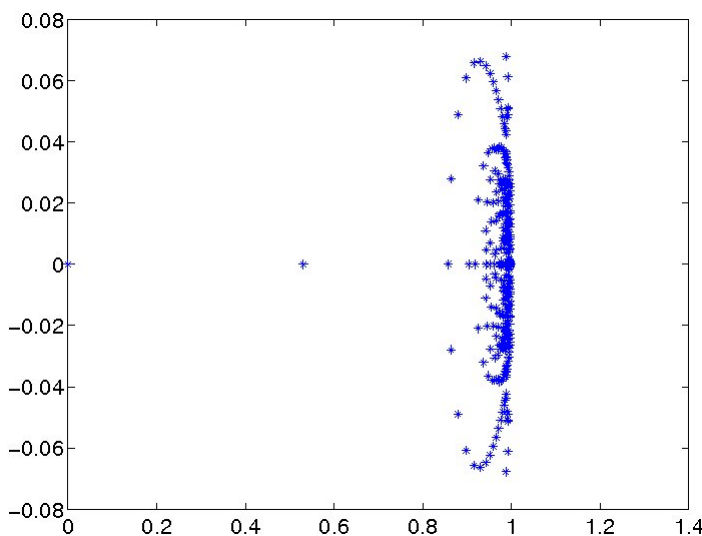

(a) $D_{M}^{-1}$

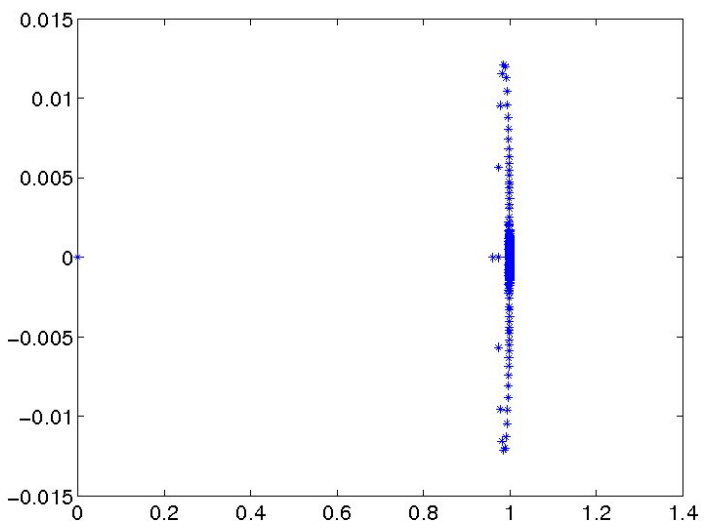

(c) $D_{\widehat{M}^{-1}}$

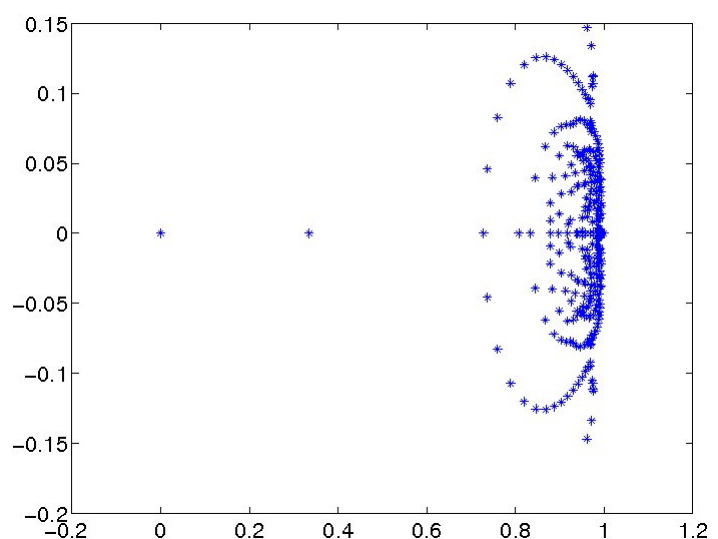

(b) $L_{M}^{-1}$

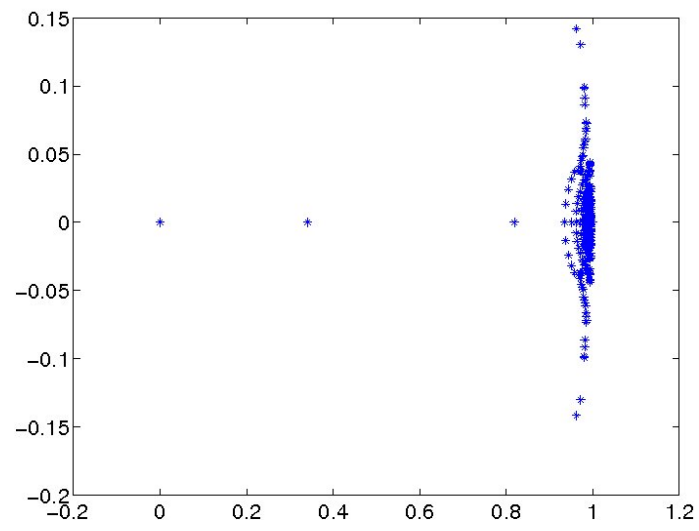

(d) $\widehat{M}^{-1}$

Figure 2: The spectrum of $\mathscr{M}_{L}^{-1} \widetilde{\mathscr{A}}, \gamma=1, v=1 / 320$

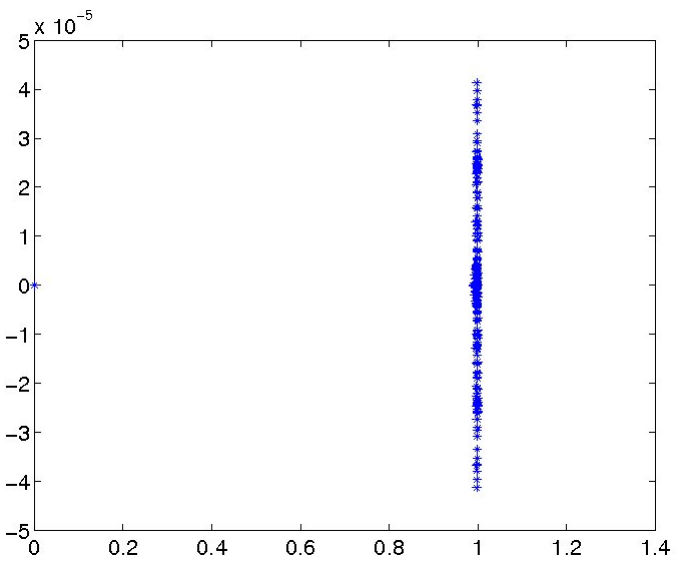

(a) $D_{M}^{-1}$

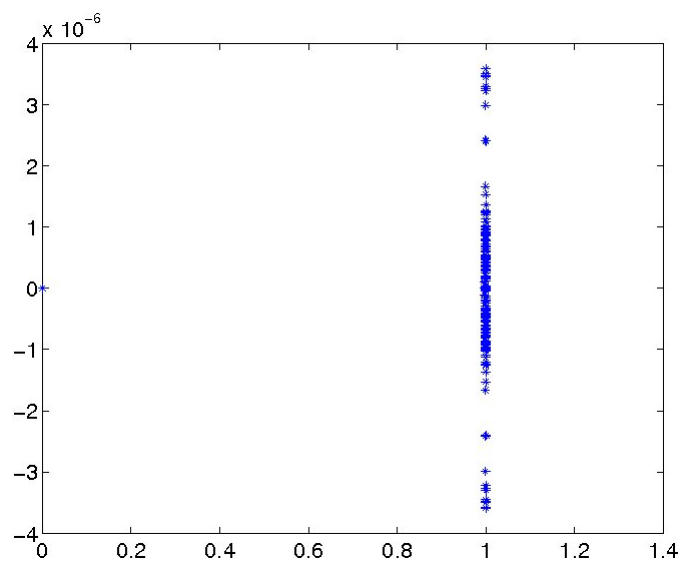

(b) $D_{\widehat{M}^{-1}}$

Figure 3: The spectrum of $\mathscr{M}_{L}^{-1} \widetilde{\mathscr{A}}, \gamma=1000, v=1$ 


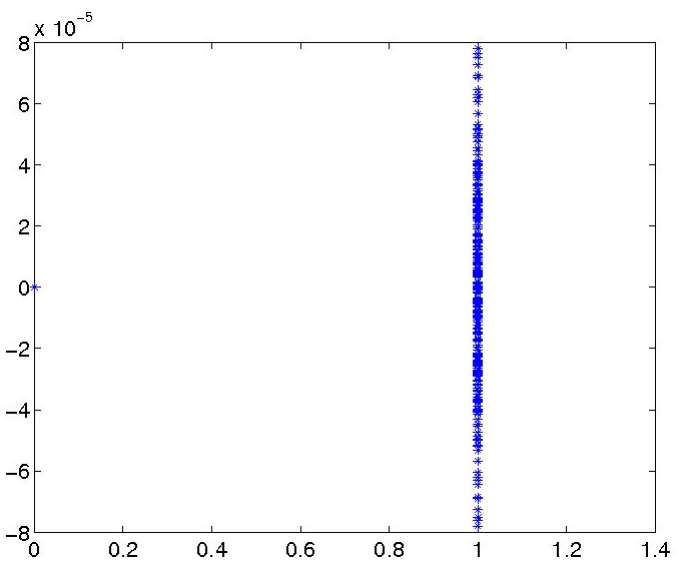

(a) $D_{M}^{-1}$

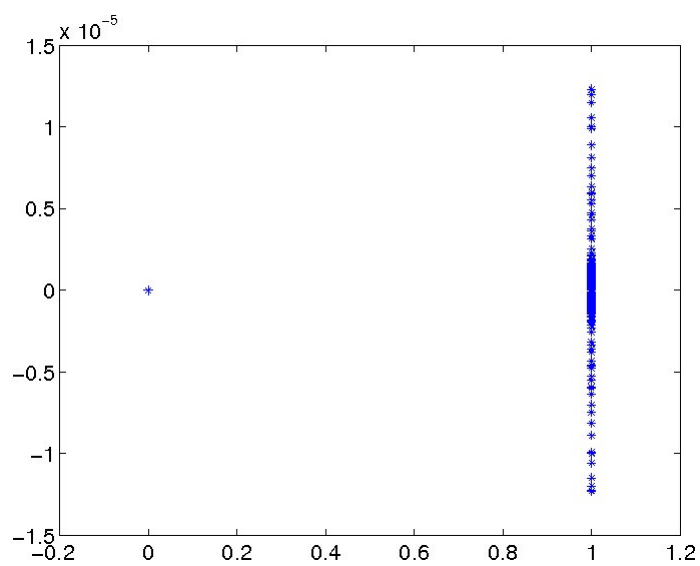

(b) $D_{\widehat{M}^{-1}}$

Figure 4: The spectrum of $\mathscr{M}_{L}^{-1} \widetilde{\mathscr{A}}, \gamma=1000, v=1 / 320$

\begin{tabular}{rccccc}
\hline & \multicolumn{5}{c}{ Iterations } \\
\cline { 2 - 5 } Size & $D_{M}$ & $L_{M}$ & $D_{\widehat{M}^{-1}}$ & $L_{\widehat{M}^{-1}}$ & $\widehat{M}^{-1}$ \\
\hline 659 & 15 & 17 & 11 & 17 & 10 \\
2467 & 15 & 18 & 10 & 15 & 10 \\
9539 & 15 & 17 & 9 & 15 & 9 \\
37507 & 14 & 17 & 9 & 15 & 9 \\
\hline \multicolumn{6}{c}{$\gamma=1000, v=1$} \\
\hline 659 & 7 & 9 & 4 & 6 & 5 \\
2467 & 5 & 7 & 4 & 4 & 4 \\
9539 & 5 & 6 & 3 & 4 & 4 \\
37507 & 4 & 6 & 3 & 4 & 4 \\
\hline \multicolumn{6}{c}{$\gamma=1, v=1 / 320$} \\
\hline 659 & 3 & 3 & 2 & 3 & 2 \\
2467 & 2 & 3 & 2 & 3 & 2 \\
9539 & 2 & 3 & 2 & 3 & 2 \\
37507 & 2 & 2 & 2 & 3 & 2 \\
\hline \multicolumn{5}{c}{$\gamma=1000, v=1 / 320$} \\
\hline 659 & 2 & 2 & 2 & 2 & 2 \\
2467 & 2 & 2 & 2 & 2 & 2 \\
9539 & 2 & 2 & 2 & 2 & 2 \\
37507 & 1 & 1 & 1 & 1 & 1 \\
\hline \multicolumn{5}{c}{$\gamma y$}
\end{tabular}

Table 2: Number of iterations to solve $\widetilde{\mathscr{A}}$, preconditioned by $\mathscr{M}_{L}$ for various approximations of $M_{p}^{-1}$ 
where $\mathbf{e}_{k}$ and $\widehat{\mathbf{a}}_{k}$ are the columns of $I_{n}$ and $\left(A-I_{n}\right)^{T}$. Then

$$
\widetilde{A}=I_{n}+\sum_{k=1}^{m+n} \mathbf{x}_{k} \mathbf{y}_{k}^{T}
$$

where $X=\left[\begin{array}{ll}B^{T} & I_{n}\end{array}\right] \subset \mathbb{R}^{n}$ and $Y=\left[\widehat{B}^{T}\left(A-I_{n}\right)^{T}\right] \subset \mathbb{R}^{n}$.

As described in $[12,13]$, for matrices in the form (29) we possess an algorithm to compute the exact inverse or an approximate inverse of $\widetilde{A}$, based on the inverse Sherman-Morrison's (ISM) formula. We advocate now its use in the AL framework.

For completeness, we include the algorithm for computing $\widetilde{A}^{-1}$, c.f., e.g., [13].

\section{Algorithm 4.1 (Algorithm ISM)}

$$
\text { for } \begin{aligned}
k & =1, \cdots, p \\
\mathbf{u}_{k} & =\mathbf{x}_{k}-\sum_{\ell=1}^{k-1} \frac{\mathbf{v}_{\ell}^{T} \mathbf{x}_{k}}{r_{\ell}} \mathbf{u}_{\ell} \\
\mathbf{v}_{k} & =\mathbf{y}_{k}-\sum_{\ell=1}^{k-1} \frac{\mathbf{y}_{k}^{T} \mathbf{u}_{\ell}}{r_{\ell}} \mathbf{v}_{\ell} \\
r_{k} & =1+\mathbf{y}_{k}^{T} \mathbf{u}_{k}=1+\mathbf{v}_{k}^{T} \mathbf{x}_{k}
\end{aligned}
$$

end

Then,

$$
\widetilde{A}^{-1}=I_{n}-U R^{-1} V^{T},
$$

where $U=\left[\mathbf{u}_{k}\right]_{k=1}^{p}, V=\left[\mathbf{v}_{k}\right]_{k=1}^{p}$ and $R=\operatorname{diag}\left(r_{1}, \cdots, r_{p}\right)$.

Remark 4.1 The algorithm is derived in $[12,13]$ for a general nonsingular matrix $A$ of the form

$$
A=A_{0}+\sum_{i=1}^{p} \mathbf{x}_{i} \mathbf{y}_{i}^{T},
$$

where $A_{0}$ nonsingular and easily invertible and $X=\left[\mathbf{x}_{k}\right]_{k=1}^{p} \subset \mathbb{R}^{n}, Y=\left[\mathbf{y}_{k}\right]_{k=1}^{p} \subset \mathbb{R}^{n}$ are two sets of vectors.

Assuming that $\mathbf{x}_{k}, \mathbf{y}_{k}, \mathbf{u}_{k}, \mathbf{v}_{k}, k=1, \cdots, p$ are such that

$$
r_{k}=1+\mathbf{y}_{k}^{T} A_{0}^{-1} \mathbf{u}_{k}=1+\mathbf{v}_{k}^{T} A_{0}^{-1} \mathbf{x}_{k} \neq 0,
$$

then Algorithm 4.1, amended with the usage of $A_{0}$, produces the inverse of $A$ as $\quad A^{-1}=$ $A_{0}^{-1}-U R^{-1} V^{T}$.

Remark 4.2 As is seen from Algorithm 4.1, $r_{k}=0$ causes a breakdown. How such situations can be handled is discussed in [13].

The following arguments, related to the ISM algorithm, warrant attention. 
(1) The algorithm consists only of scalar products and vector updates, thus, can be implemented using standard software libraries, optimized for the computer architecture, used for the numerical simulations.

(2) For dense vectors the complexity of the the algorithm is $O\left(n^{2}(n+m)=O\left(n^{3}\right)\right.$, where usually $m \approx n$, i.e., it is of the same order as that of factorizing $\widetilde{A}$, not including the cost to first explicitly form the matrix.

(3) When the vectors $X$ and $Y$ are sparse, this can be utilized in the algorithm by standard tools for sparse vector operations.

(4) As shown in $[12,13]$, various dropping rules can be applied while performing Algorithm ISM, which result in constructing approximations of $\widetilde{A}$ to be used as multiplicative preconditioners for $\widetilde{A}$ in an inner iterative procedure.

(5) The algorithm does not make any particular use of the order of the column vectors in $X$ and $Y$ and, therefore, we can pre-order them beforehand. This could be profitable in the following context. For Oseen's problem, the matrix $A$ is a sum of two matrices, $A_{L}$ and $A_{C}$, which correspond to the discretized operators $-v \Delta \mathbf{u}$ and $(\mathbf{w} \cdot \nabla) \mathbf{u}$. Thus, $\widetilde{A}=A_{L}+A_{C}+$ $\gamma B^{T} \widehat{B}$. Nothing prevents us from interchanging the matrix sum as $\widetilde{A}=A_{L}+\gamma B^{T} \widehat{B}+A_{C}$. When solving the problem in the context of the nonlinear Navier-Stokes equation, the only part of $\widetilde{A}$ which changes from one nonlinear iteration to the next is $A_{C}$. Therefore, we can update the factors $U, R$ and $V$ by recomputing only those vectors, corresponding to $A_{C}$. It is fair to note, however, that in this case $m>n$ and the recursion in Algorithm 4.1 becomes longer.

(6) Algorithm ISM is recursive, yet it possesses certain parallelization properties, for example, the vector elements could be proceeded in blocks in parallel by different processes (threads).

\section{Numerical illustrations}

We choose as a benchmark the well-known two-dimensional lid-driven cavity problem, namely, equations (1), equipped with the boundary conditions $u_{1}=u_{2}=0$ for $x=0, x=1$ and $y=0$; $u_{1}=1, u_{2}=0$ for $y=1$.

We test the following divergence-free wind vector $\mathbf{w}$, often used in the numerical tests in related articles, for example, in $[8,15]$,

$$
\mathbf{w}=\left[\begin{array}{c}
2(2 y-1)\left(1-(2 x-1)^{2}\right) \\
-2(2 x-1)\left(1-(2 y-1)^{2}\right)
\end{array}\right] .
$$

The problem is discretized using Cartesian meshes and the modified Taylor-Hood FE pair Q2isoQ2, i.e., piecewise bilinear basis functions on a mesh of size $2 h$ for the pressure and piecewise bilinear basis functions for the velocity on a mesh of size $h$, obtained by one regular refinement of the discretization mesh for the pressure. 
Table 3: Spectral properties of $M \widehat{M}^{-1}$

\begin{tabular}{ccccc}
\hline $\begin{array}{c}\text { size } \\
N(\mathscr{A}) / N(S)\end{array}$ & $\begin{array}{c}\text { mesh size } \\
h\end{array}$ & $\operatorname{Norm}\left(M \widehat{M}^{-1}\right)$ & $\operatorname{Cond}\left(M \widehat{M}^{-1}\right)$ & $\begin{array}{c}\operatorname{Eig}\left(M \widehat{M}^{-1}\right) \\
\min -\max \end{array}$ \\
\hline $659 / 81$ & $1 / 16$ & 27.3237 & 24.9329 & $1.1967-26.8132$ \\
$2467 / 289$ & $1 / 16$ & 28.0620 & 25.5948 & $1.1971-27.9666$ \\
$9529 / 1089$ & $1 / 16$ & 28.3307 & 25.8398 & $1.1971-28.3161$ \\
$37505 / 4225$ & $1 / 128$ & 28.4133 & 25.9151 & $1.1971-28.4113$ \\
$148739 / 16641$ & $1 / 256$ & 28.4363 & 25.9361 & $1.1971-28.4360$ \\
\hline
\end{tabular}

For simplicity, in the rest of this section we omit the subscript $p$ when denoting the pressure mass matrix, i.e., we use $M$ instead of $M_{p}$.

We solve the discrete problem in its $\mathrm{AL}$ form (5) (with the matrix $\widetilde{\mathscr{A}}$ ) preconditioned by the block lower-triangular preconditioner $\mathscr{M}_{L}$ or by the full block-factorized preconditioner $\mathscr{M}_{F}$. with $W^{-1}=\widehat{M}^{-1}$ and $W^{-1}=D_{\widehat{M}^{-1}}$. As a solution procedure we use the Generalized Conjugate Gradient Minimal Residual (GCG-MR) iterative method, cf., e.g., [1].

In the tables and figures below, $N(\mathscr{A})$ denotes the size of the whole coefficient matrix and $N(S)$ denotes the size of the Schur complement. Here, $h$ is the mesh-size parameter and $v$ is the viscosity parameter.

The results in Table 3 are obtained in Mat lab, while the results in Tables 4-6 are computed using a Fortran implementation of the method. There, the pivot block $\widetilde{A}$ is solved directly using the programs dgssin, dgssor, dgssfa and dgsssl from Sun Performance library (cf. [32]). The HP SL170h G6 computer server used for the Fortran codes is supported on a parallel cluster at the HPC center UPPMAX at Uppsala University, where each node consists of two Quad-core Intel@ Xeon 5520 (Nehalem 2.26 Ghz, 8MB cache) processors.

We show in Table 3 the spectral norm, the condition number and the extremal eigenvalues of the matrix product $M \widehat{M}^{-1}$. The results confirm that the spectral condition number of $M \widehat{M}^{-1}$ is mesh-independent.

Tables 4 and 5 show the iterations required for the GCG-MR to converge to a relative stopping criterion $10^{-6}$, preconditioned by $\mathscr{M}_{F}$ and $\widehat{\mathscr{M}}_{L}$. In Table 4 the preconditioned is constructed using $W^{-1}=\widehat{M}^{-1}$ and in Table 5, using $W^{-1}=D_{\widehat{M}^{-1}}$. The numerical tests confirm that the convergence does not depend on $h$ and $v$.

The condition number estimates in (24), (25), (26) and (27) are independent of mesh anisotropy, which is inherited by the preconditioners, provided that $\widetilde{A}$ is solved accurately enough. We conduct an experiment, where the mesh is initially refined along the cavity borders, introducing mesh anisotropy, measured as $h_{\max } / h_{\min }$, where $h_{\max }$ and $h_{\min }$ are the largest and smallest mesh size. The same ratio is kept through the refinement. The iterations for $W^{-1}=\widehat{M}^{-1}, \gamma=1, v=10^{-4}$ and $\mathscr{M}_{L}$ are shown in Table 6. 
Table 4: Preconditioned iterations to solve $\widetilde{\mathscr{A}}, W^{-1}=\widehat{M}^{-1}$.

\begin{tabular}{ccccc}
\hline size & mesh size & \multicolumn{3}{c}{ viscosity $v$} \\
$N(\mathscr{A}) / N(S)$ & $h$ & $10^{-2}$ & $10^{-3}$ & $10^{-4}$ \\
\hline \multicolumn{4}{c}{ Full block-factorized } & preconditioner $\mathscr{M}_{F}$ \\
\hline $659 / 81$ & $1 / 16$ & 2 & 3 & 5 \\
$2467 / 289$ & $1 / 32$ & 2 & 2 & 4 \\
$9529 / 1089$ & $1 / 64$ & 2 & 2 & 3 \\
$37505 / 4225$ & $1 / 128$ & 2 & 2 & 2 \\
$148739 / 16641$ & $1 / 256$ & 2 & 2 & 2 \\
$592387 / 66049$ & $1 / 512$ & 2 & 2 & 2 \\
\hline \multicolumn{5}{c}{ Block lower-triangular preconditioner $\mathscr{M}_{L}$} \\
\hline $659 / 81$ & $1 / 16$ & 4 & 5 & 8 \\
$2467 / 289$ & $1 / 32$ & 4 & 4 & 6 \\
$9529 / 1089$ & $1 / 64$ & 4 & 4 & 6 \\
$37505 / 4225$ & $1 / 128$ & 3 & 4 & 5 \\
$148739 / 16641$ & $1 / 256$ & 3 & 4 & 5 \\
$592387 / 66049$ & $1 / 512$ & 3 & 3 & 5 \\
\hline
\end{tabular}

Table 5: Preconditioned iterations to solve $\widetilde{\mathscr{A}}, W^{-1}=D_{\widehat{M}^{-1}}$.

\begin{tabular}{ccccc}
\hline size & mesh size & \multicolumn{3}{c}{ viscosity $v$} \\
$N(\mathscr{A}) / N(S)$ & $h$ & $10^{-2}$ & $10^{-3}$ & $10^{-4}$ \\
\hline \multicolumn{4}{c}{ Full block-factorized } & preconditioner $\mathscr{M}_{F}$ \\
\hline $659 / 81$ & $1 / 16$ & 2 & 2 & 4 \\
$2467 / 289$ & $1 / 32$ & 2 & 2 & 3 \\
$9529 / 1089$ & $1 / 64$ & 2 & 2 & 2 \\
$37505 /$ & $1 / 128$ & 2 & 2 & 2 \\
$148739 / 16641$ & $1 / 256$ & 2 & 2 & 2 \\
$592387 / 66049$ & $1 / 512$ & 2 & 2 & 2 \\
\hline \multicolumn{5}{c}{ Block lower-triangular preconditioner $\mathscr{M}_{L}$} \\
\hline $659 / 81$ & $1 / 16$ & 4 & 5 & 8 \\
$2467 / 289$ & $1 / 32$ & 4 & 4 & 5 \\
$9529 / 1089$ & $1 / 64$ & 3 & 3 & 5 \\
$37505 / 4225$ & $1 / 128$ & 3 & 3 & 4 \\
$148739 / 16641$ & $1 / 256$ & 3 & 3 & 4 \\
$592387 / 66049$ & $1 / 512$ & 3 & 3 & 4 \\
\hline
\end{tabular}


Table 6: Preconditioned iterations to solve $\widetilde{\mathscr{A}}$ with $\mathscr{M}_{L}, W^{-1}=-\widehat{M}^{-1}, v=10^{-4}, \gamma=1$

\begin{tabular}{ccccc}
\hline size & mesh size & \multicolumn{3}{c}{ mesh anisotropy } \\
$N(A) / N(S)$ & $h$ & $h_{\max } / h_{\min }=9$ & $h_{\max } / h_{\min }=49$ & $h_{\max } / h_{\min }=225$ \\
\hline $659 / 81$ & $1 / 64$ & 6 & 6 & 5 \\
$2467 / 289$ & $1 / 128$ & 6 & 5 & 5 \\
$9529 / 1089$ & $1 / 256$ & 5 & 5 & 5 \\
\hline
\end{tabular}

\section{Conclusions}

We consider the preconditioned iterative solution of Oseen type problems, reformulated using the augmented Lagrangian approach and the related tasks of choosing the method parameters $\gamma$ and $W$.

We show how the value of $\gamma$ influences the convergence rates of the outer (for $\widetilde{\mathscr{A}}$ ) and the inner (for $\widetilde{A}$ ) solution methods, namely, that small values of $\gamma$ ease the solution of inner systems, however, slow down the outer convergence, and vice versa.

Further, we consider the case when $W$ is chosen to be the pressure mass matrix and analyse various approximations of its inverse and their influence on the outer solution method. We show that the EBE-SPAI approximate of the inverse of the mass matrix is of high quality and superior that, obtained by inverting its diagonal part, however, for large values of $\gamma$, when the outer convergence is very fast, the differences between the various approximations are negligible.

We investigate also the idea of replacing $M$ by its EBE-SPAI approximation and study its effect on the block-preconditioner, compared with the pointwise diagonal approximation $D_{M}$. Even though the spectral condition number $\varkappa\left(D_{\widehat{M}^{-1}} M\right)$ turns out not to be better than $\varkappa\left(D_{M}^{-1} M\right)$, using $\widehat{M}^{-1}$ or $D_{\widehat{M}^{-1}}$ when constructing the preconditioners $\mathscr{M}_{F}$ or $\widehat{\mathscr{M}}_{L}$ leads to better clustering of the eigenvalues of $\mathscr{M}_{F}^{-1} \widetilde{\mathscr{A}}$ and $\mathscr{M}_{L}^{-1} \widetilde{\mathscr{A}}$ that when $D_{M}^{-1}$ is used to approximate $M^{-1}$. The latter affects the number of iterations when solving systems with $\widetilde{\mathscr{A}}$, in particular for small values of $\gamma$ (i.e., $\gamma=1$ ). For large values of gamma, the clustering of the eigenvalues when involving the EBE-SPAI approximation is still superior, however, provided that $\widetilde{A}$ is solved accurately, the convergence is very fast and cannot be improved any further.

Finally, we discuss the suitability of a method to compute the exact (or an approximation of the) inverse of the AL-modified pivot block $\widetilde{A}$, based on the inverse Sherman-Morrison formula. The method can be as accurate as the direct solution method for dense vectors. It has the same complexity as the direct method but it can be implemented using standard dense or sparse BLAS operations, permits partial updating in the case when the matrix changes as during a nonlinear solution procedure, and last but not least, possesses certain degree of parallelism and can be efficiently implemented on today's multithreaded/parallel computer facilities.

Acknowledgements. The work of the first two authors has been partly supported by a grant from the Swedish Research Council (VR), Finite element preconditioners for algebraic problems as arising in modelling of multiphase microstructures, 2009-2011. 
The authors thank Owe Axelsson for the valuable comments which helped to improve the presentation.

The computations were performed on resources provided by SNIC through Uppsala Multidisciplinary Center for Advanced Computational Science (UPPMAX) under Project p2009040.

\section{References}

[1] O. Axelsson, Iterative Solution Methods, Oxford University Press, 1994.

[2] O. Axelsson, Preconditioning of indefinite problems by regularization, SIAM Journal on Numerical Analysis, 16 (1979), pp. 58-69.

[3] O. Axelsson, R. Blaheta, Preconditioning of matrices partitioned in two-by-two block form: Eigenvalue estimates and Schwarz DD for mixed FEM, Numerical Linear Algebra with applications, to appear.

[4] O. Axelsson, R. Blaheta and M. Neytcheva, Preconditioning for boundary value problems using elementwise Schur complement, SIAM Journal on Matrix Analysis and Applications, 31(2009), pp. 767-789.

[5] O. Axelsson, R. Blaheta and M. Neytcheva, A black-box generalized conjugate gradient minimum residual method based on variable preconditioners and local element approximations, Technical Report 2007-033, December 2007, Department of Information Technology, Uppsala University.

[6] O. Axelsson and M. Neytcheva, Eigenvalue estimates for preconditioned saddle point matrices, Numerical Linear Algebra with applications, 13(2006), pp. 39-360.

[7] M. Benzi, G.H. Golub and J. Liesen, Numerical solution of saddle point problems, Acta Numerica, 14(2005), pp. 1-137.

[8] M. Benzi and M.A. Olshanskii, An augmented Lagrangian-based approach to the Oseen problem, SIAM J. Sci. Comput., 28(2006), pp. 2095-2113.

[9] M. Benzi, M.A. Olshanskii and Zhen Wang, Modified augmented Lagrangian preconditioners for the incompressible Navier-Stokes equations, International Journal for $\mathrm{Nu}$ merical methods in Fluids. Published online on 10 March 2010.

[10] M. Benzi, M. Tůma, A sparse approximate inverse preconditioner for nonsymmetric linear systems. SIAM J. Sci. Comput., 19 (1998), 968-994

[11] M. Benzi and Zhen Wang, Analysis of augmented lagrangian-based preconditioners for the steady incompressible Navier-Stokes equations. Math/CS Technical Report TR2010-011, June 2010. Submitted. 
[12] R. Bru, J. Cerdán, J. Marín and J. Mas, Preconditioning sparse nonsymmetric linear systems with the Sherman-Morrison formula. SIAM J. Sci. Comput., 25 (2003), 701715.

[13] R. Bru, J. Marín, J. Mas and M. Tůma, Balanced incomplete factorization, SIAM J. Sci. Comput., 30 (2008), pp. 2302-2318.

[14] E. Chow and Y. Saad, Approximate inverse techniques for block-partitioned matrices. SIAM J. Sci. Comput., 18 (1997), 1657-1675

[15] A. de Niet and F. Wubs, Two preconditioners for saddle point problems in fluid flows, International Journal for Numerical Methods in Engineering, 54 (2007), pp. 355-377.

[16] H.C. Elman and D. Silvester, Fast Nonsymmetric Iterations and Preconditioning for Navier-Stokes Equations, SIAM J. Sci. Comput, 17 (1996), 33-46.

[17] H.C. Elman, Preconditioning for the steady-state Navier-Stokes equations with low viscosity, SIAM J. Sci. Comput. 20 (1999), pp. 1299-1316

[18] H.C. Elman, D.J. Silvester, and A.J. Wathen, Finite Element and Fast Iterative Solvers: with Application in Incompressible Fluid Dynamics, Numer. Math. Sci. Comput., Oxford University Press, Oxford, UK, 2005.

[19] I. Fried, Bounds on the spectral and maximum norms of the finite element stiffness, flexibility and mass matrices. International Journal of Solids and Structures, 9 (1973), 1013-1034.

[20] I. Fried and M. Coleman, Improvable bounds on the largest eigenvalue of a completely positive finite element flexibility matrix Journal of Sound and Vibration, (283) 2005, pp. 487-494

[21] M. Fortin and R. Glowinski, Augmented Lagrangian Methods: Application to the Numerical Solution of Boundary-Value Problems, Stud. Math. Appl. 15, North-Holland, Amsterdam, New York, Oxford, 1983.

[22] T.K. Huckle, Efficient computation of sparse approximate inverses. Numer. Linear Algebra Appl, 1 (1998), 57-71.

[23] D. Kay, D. Loghin and A.J. Wathen, A preconditioner for the steady-state Navier-Stokes equations, SIAM J. Sci. Comput., 24 (2002), pp. 237-256.

[24] L. Kolotilina, Yu. Yeremin, Factorized sparse approximate inverse preconditionings. I. Theory. SIAM J. Matrix Anal. Appl., 14 (1993), 45-58.

[25] J. Kraus, Algebraic multilevel preconditioning of finite element matrices using local Schur complements, Numerical Linear Algebra with Applications, 13 (2006), 49-70. 
[26] J. Kraus, S. Margenov, Robust Algebraic Multilevel Methods and Algorithms, Radon Series on Computational and Applied Mathematics, 5, De Gruyter, 2009.

[27] M. Neytcheva, E. Bängtsson, Preconditioning of nonsymmetric saddle point systems as arising in modelling of visco-elastic problems, ETNA, 29 (2008), pp. 193-211.

[28] M. Neytcheva, He Xin and M. Do-Quang, Element-by-element Schur complete approximations for general nonsymmetric matrices of two-by-two block form, Lecture Notes in Computer Science (LNCS), 108 (2010), pp. 108-115.

[29] M.A. Olshanskii and M. Benzi, An augmented Lagrangian approach to linearized problems in hydrodynamic stability, SIAM J. Sci. Comput., 30 (2008), pp.1459-1473.

[30] M.A. Olshanskii and Y. Vassilevski, Pressure Schur complement preconditioners for the discrete Oseen problem, SIAM J. Sci. Comput., 29 (2007), pp. 2686-2704.

[31] A.J. Wathen, Realistic eigenvalue bounds for the Galerkin mass matrix, IMA Journal of Numerical Analysis 7 (1987), 449-457.

[32] Sun Performance Library Reference Manual, http://docs.sun.com/app/ docs/doc/820-2171 